\title{
ARNI verbessert bei Herzinsuffizienz auch den Glukosestoffwechsel
}

\begin{abstract}
Bei Diabetikern mit Herzinsuffizienz verbessert sich die Stoffwechsellage, wenn sie anstelle eines ACE-Hemmers mit dem "ARNI" Sacubitril/Valsartan behandelt werden, so das Ergebnis einer post-hoc-Analyse der PARADIGM-HF-Studie.
\end{abstract}

Herzinsuffizienz und Diabetes mellitus gehen häufig Hand in Hand: Man schätzt, dass etwa $40 \%$ aller Patienten mit Herzinsuffizienz auch an Diabetes leiden. Die Stoffwechselkrankheit ist dann ein unabhängiger Risikofaktor für die Progression der Herzerkrankung.

Der Angiotensin-Rezeptor-NeprilysinInhibitor (ARNI) Sacubitril/Valsartan erhöhte in früheren Studien die Insulinsensitivität bei adipösen Hypertonikern. Ob die Substanz generell den Glukosestoffwechsel günstiger beeinflusst als ein (stoffwechselneutraler) ACE-Hemmer, wurde jetzt anhand des DiabetikerKollektives in der PARADIGM-HFStudie untersucht. Zur Erinnerung: In dieser Studie hatte Sacubitril/Valsartan bei Patienten mit systolischer Herzinsuffizienz die Morbidität und Mortalität günstiger beeinflusst als Enalapril.

Bei 3778 der 8399 Studienpatienten war ein Diabetes bekannt. Sie waren bezüglich Anzahl und Risikokonstellation gleichmäßig auf die Sacubitril/Valsartanund Enalapril-Gruppe verteilt.

Autoren aus Boston verglichen posthoc die Stoffwechseleinstellungen in beiden Gruppen und stellten fest, dass innerhalb des ersten Therapiejahres der $\mathrm{HbA}_{1 \mathrm{c}}$-Wert in der ARNI-Gruppe um $0,26 \%$ abgefallen war und in der Enalapril-Gruppe um 0,16\%. Der Unterschied blieb über die gesamte Studienzeit von drei Jahren bestehen und war statistisch signifikant.

114 (7\%) der Patienten in der ARNIGruppe wurden in dieser Zeit Insulinpflichtig, im Vergleich zu 153 (10\%) in der Enalapril-Gruppe. Auch bezüglich des Beginns einer Therapie mit oralen Antidiabetika zeigte sich ein entsprechender Unterschied.

\section{Therapie nach Herzinfarkt}

\section{Behandlung der erektilen Dysfunktion mit geringerer Mortalität assoziiert}

Patienten, die sich nach einem Herzinfarkt gegen erektile Dysfunktion (ED) behandeln lassen, haben bessere Überlebenschancen als vergleichbare Patienten ohne ED-Medikation, wie Ergebnisse einer schwedischen Studie zeigen.

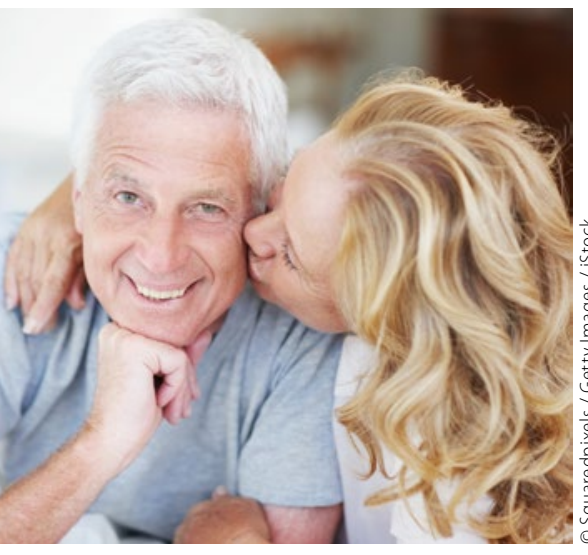

Gesündere Männer bitten vermutlich eher um ein Rezept für eine ED-Medikation.
Männer, die einen Herzinfarkt erlitten haben und danach gegen ED behandelt werden, leben im Mittel länger als Infarktpatienten ohne ED-Therapie. Dies ist das Ergebnis einer schwedischen Kohortenstudie, in der Daniel Andersson und sein Forscherteam 43.145 schwedische Männer untersucht haben, die zwischen 2007 und 2013 erstmals wegen eines Herzinfarkts in stationärer Behandlung gewesen waren.

In der Nachbeobachtungszeit von im Mittel 3,3 Jahren lag die Sterblichkeit bei Männern, die gegen ED behandelt wurden, um 33\% niedriger als für jene, die keine ED-Medikamente einnahmen.
Auch das Risiko größerer Herzkomplikationen war um $21 \%$ geringer. Im Vergleich zwischen verordnetem Alprostadil und PDE-5-Hemmern waren aber nur Letztere mit einer verminderten Mortalität verbunden.

Wie Andersson und Kollegen einräumen, lässt sich aus diesen Assoziationen keine Kausalität herleiten. Denn es ist von einer systematischen Verzerrung auszugehen, da gesündere Männer mit höherer Wahrscheinlichkeit um ein Rezept für ein ED-Medikament bitten. In der Studienpopulation waren die Männer mit EDBehandlung auch jünger und gesünder als die Männer ohne solche Arzneien.

Dr. Robert Bublak

Andersson DP et al. Heart 2017, Online 09 März. doi: 10.1136/heartjnl-2016-310746 